\title{
Development of Dipeptide Transport in the Intestinal Mucosa of Rabbits
}

\author{
STEFANO GUANDALINI ${ }^{(32)}$ AND ARMIDO RUBINO \\ Child Health Institute, 2nd School of Medicine, University of Naples, Italy
}

\section{Summary}

The uptake of $0.5 \mathrm{mM}$ glycyl-L-proline (Gly-Pro), a substrate which has affinity for the dipeptide specific carrier system and is not hydrolyzed in the brush border, was studied in vitro in jejunum and ileum of rabbits from the 25th day of gestational age into adulthood. For comparison, uptake of $0.5 \mathrm{mM}$ glycine was studied too. Results show that influx of Gly-Pro is present from the earliest age studied and show a steep prenatal increase and peaks at birth, with values, in the newborn jejunum, of $45.2 \pm 3.3 \mu$ moles/ ghr (mean \pm S.E.). Thereafter, the uptake slowly declines toward the adult level of $3.8 \pm 0.5 \mu \mathrm{moles} / \mathrm{ghr}$. A similar pattern was present in the ileum. On the contrary, free glycine uptake shows no statistically significant change during the whole period studied.

The characteristics of the Gly-Pro influx process have been studied in the period of its peak activity (1-to 6-day-old rabbits) in both jejunum and ileum. The process is not inhibited by a 40 -fold excess of glycine, proline, phenylalanine, leucine, lysine, glutamic acid, while it is strongly inhibited by a 10-fold excess of the dipeptides leucyl-leucine, alanyl-proline, glycyl-leucine and lysylleucine. A large excess of Gly-Pro has no inhibitory effect on the uptake of free glycine. The kinetic constants for Gly-Pro uptake are: $V \max =126.7 \pm 26.4 \mu$ moles/ghr and $K t=0.98 \pm 0.26 \mathrm{mM}$ in the jejunum and $V \max =59.6 \pm 9.2$ and $\mathrm{Kt}=0.70 \pm 0.10$ in the ileum. The removal of $\mathrm{Na}$ from the incubation and preincubation medium approximately halves the Vmax in both segments, leaving unchanged the affinity constants.

These results indicate that a very efficient dipeptide influx process occurs in the newborn rabbit. This process displays many characteristics in common with that described in the adult: it is not shared by amino acids free in the lumen; has a broad specificity for dipeptides and shows the same pattern of $\mathrm{Na}$ dependence. However, its maximal velocity is in the newborn much higher than in the adult.

Therefore, (1) the developmental patterns of the transport systems for Gly-Pro and for free glycine are different; this provides ontogenetic evidence for the distinction between dipeptide and free amino acid carrier systems; and (2) dipeptide uptake plays a predominant role in the physiology of the protein absorptivedigestive processes of the youngster.

\section{Speculation}

Dipeptide uptake is likely to play a major role in the final stages of protein absorption in the youngster. Thus, wherever proteic oral supplementation is indicated in the newborn or young infant, the administration of free amino acids does not appear to be physiologically appropriate.

The final products of intraluminal digestion of protein are low molecular weight peptides and free amino acids (6). A substantial body of evidence accumulated in the adult animal has shown that di- and tripeptides can be transported into the intestinal cells by a specific carrier system which is different from those involved in the transport of free amino acids (reviewed in Ref. 19). On the other hand, they can also be hydrolyzed at the brush border membrane, with subsequent entry of the released amino acids.

We had previously shown (24) that both ways do occur also in the small intestine of the newborn rabbit, the transport of intact dipeptide being quantitatively approximately as important as the other pathway. We thus moved to investigate, in the developing rabbit, the carrier system for dipeptides, with these major aims: (1) provide a biologic characterization of the system as for substrate specificity, Na dependence and kinetic constants; (2) define its developmental pattern as compared to that of the uptake of a free neutral amino acid, as to provide an ontogenetic basis for the distinction between the two systems; (3) assess, in the youngster, the relative importance of dipeptide vs. free amino acid uptake.

Therefore, the uptakes of glycyl-proline labeled in the glycine residue and, for comparison, that of free glycine, have been studied in vitro in jejunal and ileal segments of fetal, newborn, suckling, and adult rabbits.

Glycyl-proline has been chosen as a substrate, because it has affinity for the dipeptide carrier system but is not hydrolyzed in the brush border, its hydrolysis being accomplished entirely intracellularly $(20,29)$.

\section{MATERIALS AND METHODS}

\section{MEASUREMENT OF UNIDIRECTIONAL INFLUX OF SUBSTRATES} FROM LUMEN INTO THE EPITHELIUM

New Zealand White rabbits were used: fetuses (from the 25 th to the 30th day of gestational age), newborns ( 1 to 6 days old), suckling and weaned (at the $10 \mathrm{th}, 20 \mathrm{th}, 30 \mathrm{th}, 40 \mathrm{th}$, and 50 th day of postnatal age), and adults (older than 3 months).

For studies in the fetuses, the pregnant rabbit was killed by cervical dislocation and the fetuses, quickly removed from the uterus, were then killed by decapitation.

The newborns had always been fed by their mothers (as judged by the presence of milk in the stomach), and were also killed by decapitation.

The animals beyond 10 days of postnatal life were killed by cervical dislocation.

In all cases, the intestinal segment under study was quickly excised, opened along the mesenteric border, and rinsed free of intestinal contents with a Ringer solution.

In the fetuses, studies were done on the proximal and distal third of the small intestine, heretofore referred to as jejunum and ileum, respectively. In the newborns and thereafter, the small intestine (distal to the ligament of Treitz) was divided in five segments: the first two-fifths referred to as proximal and distal jejunum, and the last two-fifths referred to as proximal and distal ileum; the middle fifth was discarded.

Influx measurements were then carried out in the adult animals according to the technique previously described (23). In all the animals of younger age, the same basic technique was followed, with some appropriate modifications. In these ages, the following procedure was used: the segment under study was preincubated for $30 \mathrm{~min}$ in a Ringer solution having, unless otherwise indicated, the following composition: $\mathrm{NaCl}, 140 \mathrm{mM} ; \mathrm{KHCO}_{3}, 10 \mathrm{mM}$; 
$\mathrm{K}_{2} \mathrm{HPO}_{4}, 1.2 \mathrm{mM} ; \mathrm{KH}_{2} \mathrm{PO}_{4}, 0.2 \mathrm{mM} ; \mathrm{CaCl}_{2}, 1.2 \mathrm{mM}$; and $\mathrm{MgCl}_{2}$, $1.2 \mathrm{mM}$ (pH 7.2). Preincubation was carried out at $37^{\circ} \mathrm{C}$ and with bubbling $\mathrm{O}_{2}-\mathrm{CO}_{2}$ (95 to $5 \%$ ).

Pieces (100 to $300 \mathrm{mg}$ ) of the intestinal segment were then individually placed in an Erlenmeyer flask containing the test solution. This was a Ringer solution of identical composition, but containing the radioactively labeled compound under study (either (Glycyl $-{ }^{14} \mathrm{C}$ ) glycyl-proline or ${ }^{3} \mathrm{H}$-glycine) and inulin (labeled with either ${ }^{3} \mathrm{H}$ or ${ }^{14} \mathrm{C}$ ) as a marker of the extracellular space. The incubation in the test solution described was also carried out at $37^{\circ} \mathrm{C}$ and with gassing $\mathrm{O}_{2}-\mathrm{CO}_{2}(95$ to $5 \%$ ). After 50 to $60 \mathrm{sec}$, the piece was quickly removed, rinsed in cold $0.3 \mathrm{M}$ Mannitol solution, blotted on filter paper, placed in $2 \mathrm{ml}$ of $10 \%$ thrichloroacetic acid (TCA), and weighed. After homogeneization by means of an Ultra-Turrax tissue homogenizer, tissues were centrifuged at 5000 $\times g$ for $8 \mathrm{~min}$ and aliquots of the supernatants were then added to Bray's solution and assayed for radioactivity in a liquid scintillation counter. Aliquots (50 to $100 \mu \mathrm{l}$ ) of the media were similarly counted; the total content of $10 \%$ TCA in all scintillation vials was kept constant, so as to minimize quenching variations among different samples.

Calculations were done accordingly to the described procedure (23) and data expressed for animals of all ages on the basis of weight.

With the experimental procedure described above, both the luminal and the serosal sides of the intestine were exposed to the incubation medium. However, preliminary experiments were done under conditions in which only the serosal side was exposed: both glycyl-proline and glycine uptakes were always less than $5 \%$ of the "total" uptakes, thus allowing to assume that more than $95 \%$ of the measured influx is due to mucosal uptake alone.

Also in preliminary experiments (data not shown) it was demonstrated that: $(a)$ more than $98 \%$ of the total tissue radioactivity was present in the supernatant after the extraction procedures; and (2) tissue uptakes of both, (glycyl- ${ }^{14} \mathrm{C}$ ) glycyl-L-proline (GlyPro) and ${ }^{3} \mathrm{H}$-glycine (Gly) after correction for the inulin space, were linear with time for at least the first $60 \mathrm{sec}$ and extrapolate to the origin.

It can, therefore, be assumed that under these conditions, initial rates of unidirectional influx of Gly-Pro and Gly are measured.

\section{EFFECT OF INHIBITORS AND COMPUTATION OF KINETIC} CONSTANTS

The inhibitor effects were tested by adding the test substance to the incubation medium of one piece, while the adjacent segment (in which an amount of mannitol equimolar to the inhibitor was added) served as control. To determine the kinetic constants, four substrate concentrations were tested in each animal. To eliminate any difference due to intestinal gradients (within both jejunum and ileum) the concentrations were randomized with respect to the localization of the single intestinal piece. Values for maximal influx (Vmax) and apparent affinity constant $\left(\mathrm{K}_{\mathrm{t}}\right)$ were determined, respectively, from the reciprocal of the $Y$ intercept and from the slope times the $\mathrm{Y}$ intercept of the least squares regression line of a reciprocal plot of influx/concentration; i.e., from the following linear transformation of the Michaelis-Menten equation:

$$
\frac{1}{V}=\frac{1}{V \max }+\frac{K_{t}}{V \max } \times\left(\frac{1}{S}\right)
$$

\section{MATERIALS}

(Glycyl-14C)glycyl-L-proline, $\left({ }^{3} \mathrm{H}\right)$ inulin, $\left({ }^{3} \mathrm{H}\right)$ Glycine and inulin-(14C)carboxylic acid were obtained from the Radiochemical Centre-Amersham, Buckinghamshire, England.

L-Alanyl-L-proline, glycyl-L-leucine, glycyl-L-proline, L-prolylglycine, L-leucyl-L-leucine, L-lysyl-L-leucine, glycine, L-glutamic acid, L-leucine, L-lysine, L-phenylalanine, L-proline were purchased from Sigma Chemical Company, Saint Louis, MO. All other reagents were analytical grade.
Statistical evaluation of the data was done by Student's $t$ test for unpaired or, where appropriate for paired values. All values are expressed as means \pm 1 S.E.

\section{RESULTS}

\section{STUDIES IN THE NEWBORN}

Uptake of (Glycyl- $\left.{ }^{14} \mathrm{C}\right)$ Glycyl-L-proline and of ${ }^{3} \mathrm{H}$-Glycine by Small Intestine of Newborn Rabbit. The uptakes of $0.5 \mathrm{mM}$ GlyPro and of $0.5 \mathrm{mM}$ Gly by segments of jejunum and ileum of newborn ( 1 to 6 days old) and for comparison of adult animals are reported in Table 1. Gly-Pro is taken up by the small intestine of newborns in much larger amount than an equimolar amount of Gly; this is also true, though to a lesser extent, in the jejunum of the adult. Furthermore, in both the intestinal segments tested, Gly-Pro uptake is much larger in the newborn than in the adult animal (all these differences being statistically highly significant). Finally, a different distribution pattern for Gly-Pro and Gly uptake is seen along the small intestine of the newborn: sites of maximal uptake of the dipeptide occur proximally, while free glycine has its highest influx levels in the distal ileum.

It is of interest that the same conclusions can be drawn also when the values are expressed on the basis of tissue area.

Effects of $\alpha-L$-Amino Acids and Peptides on Gly-Pro Influx. To investigate the specificity of the Gly-Pro influx, we have measured it under conditions in which either a 40-fold excess of free amino acids or a 10-fold excess of dipeptides was present. As it is indicated in Table $2 A$, none of the tested amino acids (and among them Glycine and Proline) showed any significant inhibition of Gly-Pro influx in either jejunum or ileum. The presence of $5 \mathrm{mM}$ Leu-Leu, Ala-Pro, Gly-Leu or Lys-Leu, on the contrary, substantially and significantly inhibited $0.5 \mathrm{mM}$ Gly-Pro uptake in both segments. In the jejunum, the effect of a 40 -fold excess of L-prolylglycine (a dipeptide lacking a free amino group) was also tested: similarly to what already found in the adult animal (23), it failed to produce any inhibition of Gly-Pro uptake.

These data indicate that the Gly-Pro transport system in the newborn animal is not shared by free amino acids, while possessing a broad specificity for dipeptides.

Effect of Gly-Pro on Gly Uptake. To further verify the independence of the uptake processes for Gly-Pro and free Gly, the effect of a 40-fold excess of Gly-Pro on the uptake of ${ }^{3} \mathrm{H}$-glycine was then tested. The results (Table $2 B$ ) show that the influx of free Gly is not inhibited by a large excess of Gly-Pro. Thus, also in the newborn animal the carrier responsible for the uptake of free Gly has no affinity for Gly-Pro.

These data, along with those presented in the preceding section, provide sufficient evidence for the distinction of the dipeptide transport system from the amino acid transport systems in the developing animal.

Kinetic Constants and Sodium Dependence of Gly-Pro Influx. While studies in the ileum of adult rabbit suggest the presence of two carrier processes for Gly-Pro (23), one with a high $\mathrm{K}_{\mathrm{t}}$ and a high Vmax, and the other with a low $\mathrm{K}_{\mathrm{t}}$ and a low Vmax, evidence for only the latter has been obtained in the jejunum of adults (25).

We have investigated, in the newborn, the kinetic characteristics of the Gly-Pro influx process in the substrate concentration range of 0.05 to $1.6 \mathrm{mM}$, in both jejunum and ileum.

Results (Fig. $1 A$ and $B$, lower lines) show that over this concentration range, and in presence of $140 \mathrm{mM} \mathrm{NaCl}$, the initial rate of unidirectional influx of Gly-Pro follows, in both intestinal segments, a saturable pattern. In other words, on increasing the substrate concentration, the substrate uptake increases until a plateau is reached, according to Michaelis-Menten type of kinetics. Results are shown in Figure 1 as double reciprocal plots, i.e., uptake $^{-1} /$ concentration $^{-1}$. The apparent kinetic constants of this process are: $\mathrm{Vmax}=126.7 \pm 26.4 \mu$ moles $/ \mathrm{g} \mathrm{hr}, \mathrm{K}_{\mathrm{t}}=0.98 \pm 0.26$ $\mathrm{mM}$ in the jejunum and $59.6 \pm 9.2$ and $0.70 \pm 0.1$, respectively, in the ileum. While the two $V \max$ are different $(P<0.05)$, no 
Table 1. Uptake of $0.5 \mathrm{mM} \mathrm{GLY-PRO}$ and of $0.5 \mathrm{mM}$ GLY by the small intestine of newborn and adult rabbits ${ }^{1}$

\begin{tabular}{clcccc}
\hline & & \multicolumn{2}{c}{ Intestinal segment } \\
\cline { 3 - 5 } Age & Substrate & Proximal jejunum & Distal jejunum & Proximal ileum & Distal ileum \\
\hline \multirow{2}{*}{ Newborn } & Gly-Pro & $45.2 \pm 3.3(14)$ & $42.9 \pm 2.9(12)$ & $25.4 \pm 1.5(13)$ & $21.1 \pm 1.8(16)$ \\
& Gly & $3.3 \pm 0.1(6)$ & $3.1 \pm 0.2(5)$ & $3.3 \pm 0.6(5)$ & $5.7 \pm 1.1(6)$ \\
& & & & $6.1 \pm 0.6(11)$ \\
Adult & Gly-Pro & $3.8 \pm 0.5(11)$ & & $6.5 \pm 0.9(6)$ \\
\hline
\end{tabular}

'Values are $\mu$ moles/ghr, mean \pm S.E. Number of animals is given in parentheses. In the newborn, in all segments, Gly-Pro uptake is significantly $(P<0.001)$ higher than Gly uptake. In the adult, Gly-Pro is also significantly $(P<0.01)$ higher than Gly uptake only in the proximal jejunum. In the newborn as compared to the adult, Gly-Pro uptake is significantly $(P<0.001)$ higher in all segments, while Gly uptake is significantly $(P<0.05)$ higher in the jejunum but not different in the ileum.

Table 2. Effects of inhibitors on $\left({ }^{14} \mathrm{C}\right) \mathrm{GLY}$-PRO or $\left({ }^{3} \mathrm{H}\right)$ glycine influx ${ }^{1}$

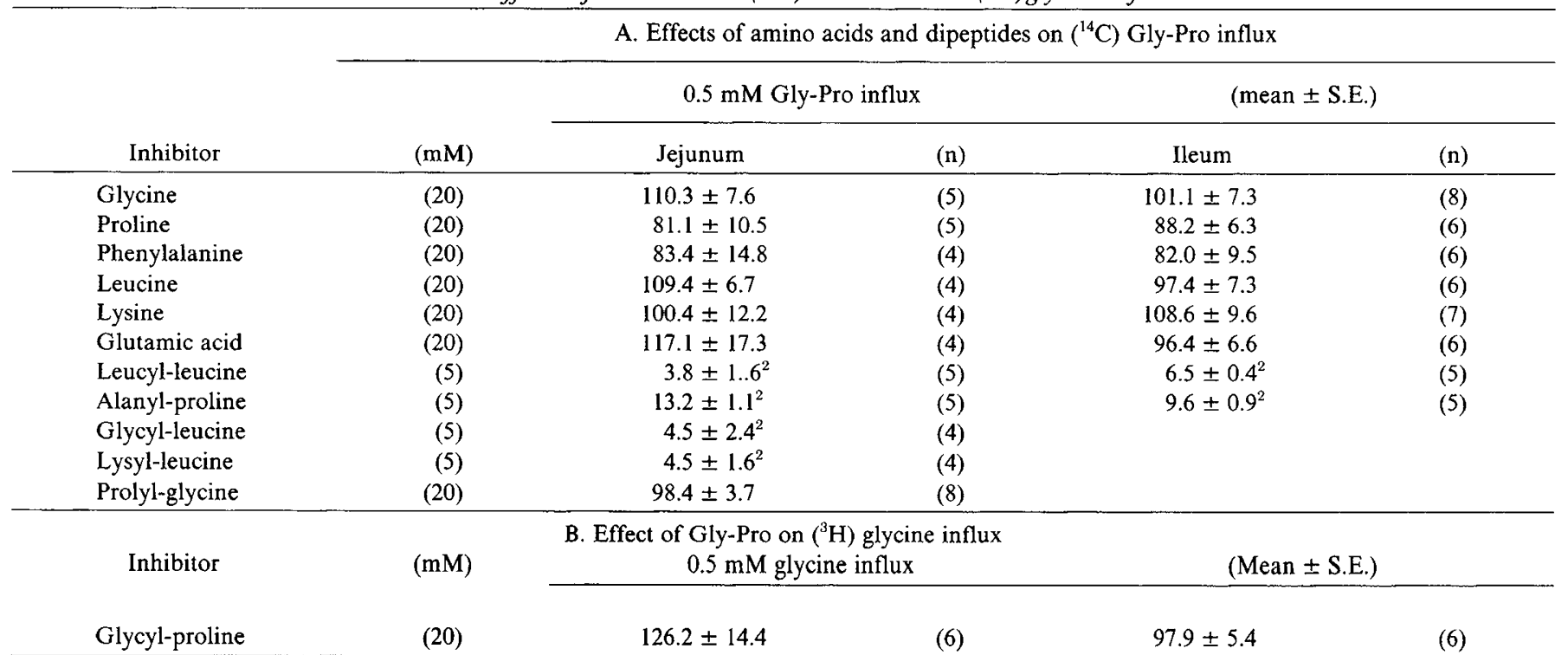

\footnotetext{
${ }^{1}$ Influx in presence of inhibitors is expressed as percent of control influx. Control and inhibited tissues were immediately adjacent segments and the proximal-distal sequence was alternated in each experiment. The data were treated as pairs within each experiment. Inhibitor concentrations and number of animals are given in parentheses.
}

${ }^{2} P<0.0001$.

statistically significant difference exists between the affinity constants.

The effect of $\mathrm{Na}^{+}$removal on these kinetic characteristics has been studied, too. The results, also shown in Figure $1 A$ and $B$, (upper lines) indicate that the total substitution of choline for $\mathrm{Na}$ (both in the preincubation and in the incubation media) severely affects the efficiency of the Gly-Pro influx process. In both jejunum and ileum the decrease in Gly-Pro uptake is due to a significant reduction of $V \max$, which decreases to $50.6 \pm 12.2$ $\mu$ moles $/ \mathrm{g} \mathrm{hr}$ in the jejunum and to $29.0 \pm 7.1$ in the ileum. The affinity constants of the process remained essentially unchanged both in the jejunum (from $0.98 \pm 0.26 \mathrm{mM}$ in presence of 140 $\mathrm{mM} \mathrm{Na}$ to $0.83 \pm 0.15$ in its absence) and in the ileum (from 0.70 $\pm 0.1 \mathrm{mM}$ in presence of $140 \mathrm{mM} \mathrm{Na}$ to $0.61 \pm 0.25$ in its absence).

\section{DEVELOPMENTAL STUDIES}

Developmental Pattern of Gly-Pro and Free Gly Uptake Processes. To define more accurately the age dependence of the increased Gly-Pro influx rates in the newborn as compared to the adult animal, as well as to detect possible differences in the developmental patterns of their uptake processes, we have measured $0.5 \mathrm{mM}$ Gly-Pro and $0.5 \mathrm{mM}$ free Gly influx in the small intestine of rabbits since the 25 th day of gestational age to the 50 th postnatal day. The results are reported in Figure 2 for jejunum and Figure 3 for ileum. In these figures, corresponding values found in the adult are shown too. Gly-Pro influx showed in both jejunum and ileum, a gradual increase from the earliest fetal period studied, with a striking perinatal peak. The uptake of Gly-Pro did not show any significant change when measured at 2 $\mathrm{hr}$ after birth and every $12 \mathrm{hr}$ thereafter for the first 3 days (newborns from four different litters tested longitudinally, data not shown). After the first 6 days of postnatal life, Gly-Pro uptake declined steadily, reaching adult values at the age of about 50 days. In contrast, free Gly uptake showed no well-defined prenatal increase; furthermore, in the ileum, it remained constant throughout the period studied, while in the jejunum it was only slightly bigger in the younger animal.

It is of interest to note that in both jejunum and ileum, uptake of Gly-Pro remains remarkably higher than Gly uptake in the fetal, newborn, and suckling rabbits.

Studies done on Gly-Pro dipeptidase activity in homogenates of whole intestinal mucosa of developing rabbits (22) had shown an essentially analogous developmental pattern in both small intestinal segments.

\section{DISCUSSION}

The evidence for the distinction between the small intestinal carrier system for dipeptides and those for free amino acids has rested so far mainly on the two following pieces of evidence: (1) studies in man had shown that, in diseases characterised by the lack of a specific amino acid carrier system, the absorption of the "affected" amino acid is intact if this is presented to the mucosa in the peptide-bound form $(5,15)$; (2) dipeptides do not compete with their constituent amino acids in the free form for transport, while they do compete with one another $(1-3,7,8,23)$. 

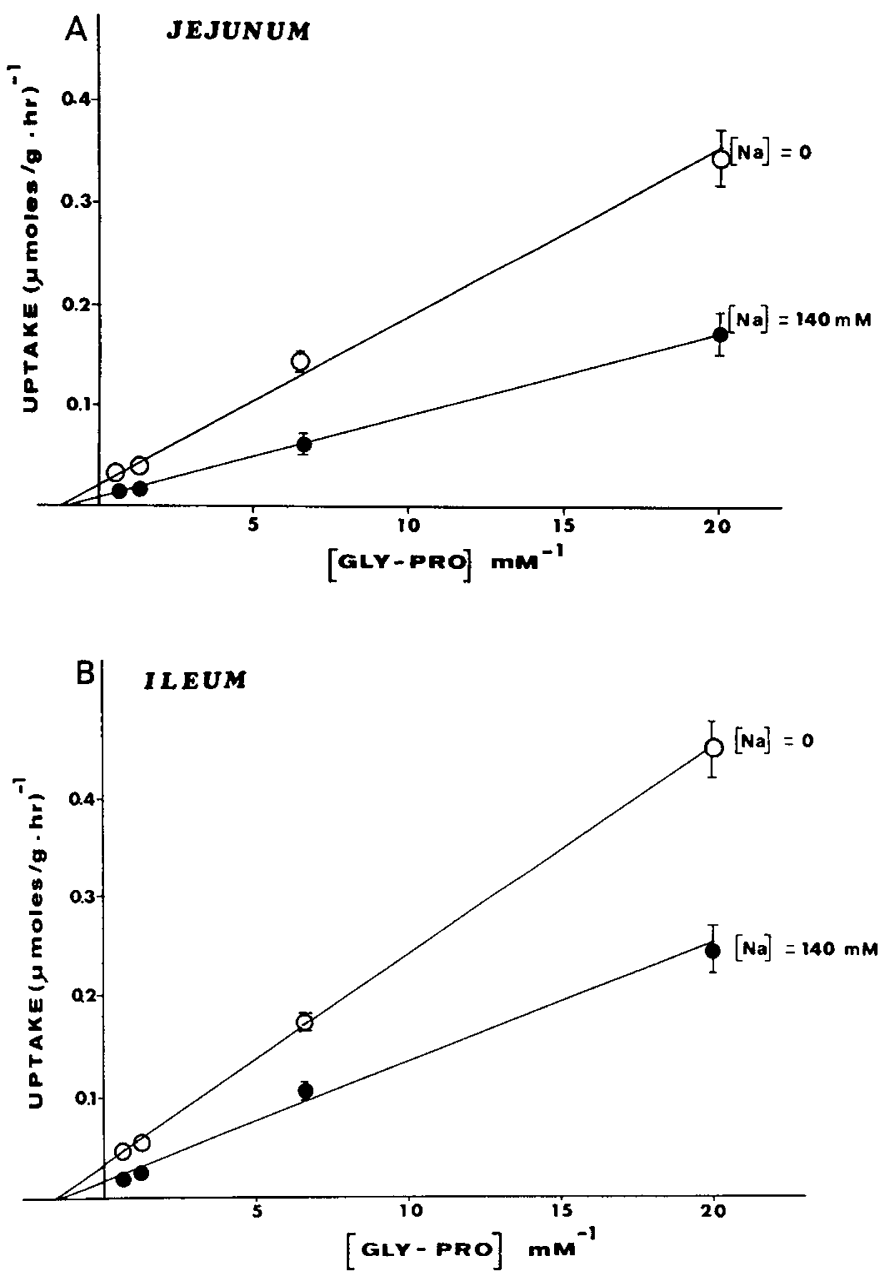

Fig. 1. Kinetic characteristics of the Gly-Pro influx process in the small intestine of the newborn rabbit. Reported here are double reciprocal plots of Gly-Pro influx at various substrate concentrations. Brackets indicate \pm S.E. $A$ Jejunum: $\bullet$, in the presence of $140 \mathrm{mM} \mathrm{Na}$; $O$, in the absence of Na. $B$ lleum: - in the presence of $140 \mathrm{mM} \mathrm{Na}$; $\mathrm{O}$, in the absence of $\mathrm{Na}$. Removal of $\mathrm{Na}$ significantly $(P<0.05)$ affected the $\mathrm{Vmax}$ in both segments, while having no significant effect on $\mathrm{K}_{\mathrm{t}}$.

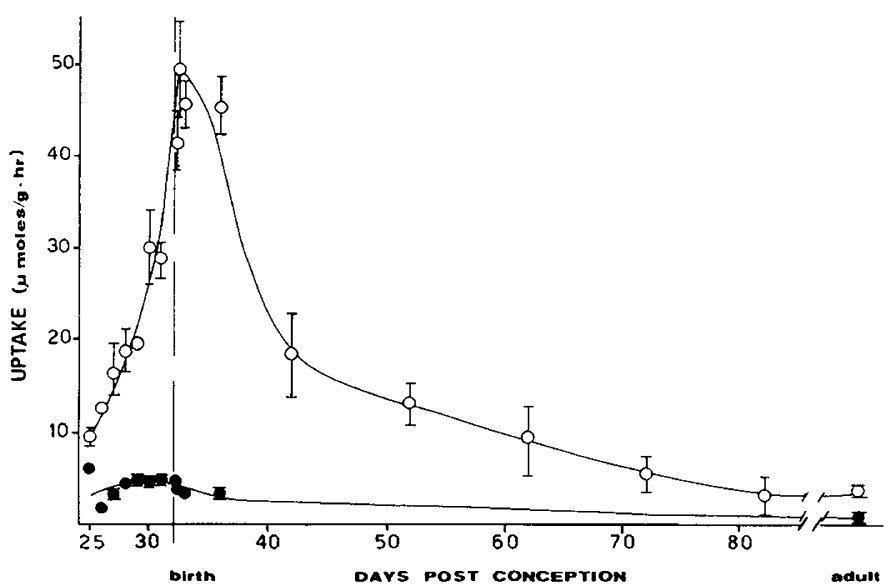

Fig. 2. Uptake of Gly-Pro $(O)$ and of free Gly $(\bullet)$ in the jejunum of fetal, newborn, and suckling rabbits. Brackets indicate $S$. E. Both substrates were $0.5 \mathrm{mM}$.

This paper now provides another line of evidence on which the distinction between the two systems can further rest: their different ontogenetic pattern. In fact, the developmental pattern of the carrier system for the glycine residue of glycylproline shows, in both jejunum and ileum, a definite prenatal increase with a sharp

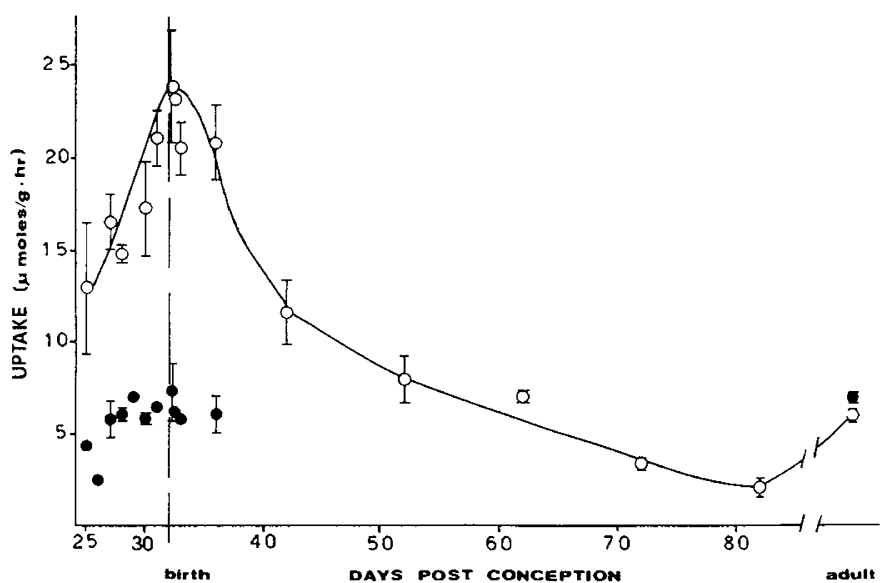

Fig. 3. Uptake of Gly-Pro $(O)$ and of free Gly $(O)$ in the ileum of fetal, newborn, and suckling rabbits. Brackets indicate S.E. Both substrates were $0.5 \mathrm{mM}$.

perinatal peak and a subsequent decline, while these characteristics are not shared by the uptake process for free glycine: this persists essentially unchanged in the whole period studied in the ileum, and in the jejunum only shows slightly higher values in the neonatal age as compared to the adult.

The main characteristics of the dipeptide carrier system studied at its peak, during the newborn age, can be summarized as follows: (1) it is saturable; (2) its maximal velocity is Na dependent; (3) it is not inhibited by the presence of a large excess of the constituent amino acids when presented free to the mucosa; (4) it has a broad specificity for different dipeptides.

These characteristics are analogous to those found in the ileum of the adult animal (23). Therein, evidence was also found for a second system with a higher $\mathrm{Vmax}$ and a higher $\mathrm{Kt}$ : whether or not this system, which has not been looked for in greater detail, is present also in the newborn, we do not know. As for the $\mathrm{Na}$ dependence of the system, it should be noted that we do not know which mechanisms underlie the role played by $\mathrm{Na}$ in the Gly-Pro influx process; however, from our own results in the adult animal (13) it would not appear that a coupled influx is involved as in the case of glucose or neutral amino acids.

It is interesting to note that, although the high affinity dipeptide uptake system shows so many similarities between the adult and the newborn animal, a striking difference exists concerning the maximal velocity. In fact, this is in the newborn greater by a factor of about 13; given the analogous Kt's of the systems, it is evident that dipeptide uptake is a process definitely more efficient in the newborn than in the adult. It has to be stressed that this conclusion is essentially true whether the data are expressed on the basis of weight or on the basis of area, thus excluding that differences in tissue weight per surface area unit between newborn and adult are responsible for this observation.

To support this conclusion further, it can be noted that ileal uptake of free Gly does not show any variation in the whole developmental period studied, being not significantly different between newborn and adult.

The higher Gly-Pro influx rate found in the newborn and suckling rabbits, being accounted for by a bigger maximal velocity, may be due either to a larger number of carrier sites present per unit area in the younger animals, or to a faster translocation velocity of the Gly-Pro-carrier complex across the brush border (or both). Our data do not allow to distinguish between these two alternatives. Other instances are known, where a different Vmax but similar affinity constant in transport processes between young and adult animals have been demonstrated. In rat $(11,21,30)$ and rabbit (9) intestine, the transport systems studied showed their highest capacity perinatally, and subsequently they progressively declined to the adult values, similarly to what we report herein for dipeptide uptake. It is of interest to note that the fetal period of fast-paced increase in Gly-Pro uptake activity is coincident (9) with the rapid development of villous and microvillous structure. 
Yet, the simultaneously measured uptake of free Gly does not show any clear prenatal increase, thus suggesting that the increase in Gly-Pro uptake is not merely due to the increasing amount of brush border membrane per unit area associated to tissue maturation.

Various factors may affect, theoretically, the activity of a transport system. Hormones, plasma levels of the nutrient, feeding habits, etc., all might have a role. As for dietetic influences, for instance, an effect of semistarvation has been documented on the in vitro transport of amino acids $(26,31)$ and dipeptides $(14,26)$.

In this report, we have not investigated the mechanisms responsible for either the progressive prenatal increase or the steadily postneonatal decline in the influx of Gly-Pro. While studies to answer this question are needed, it may, nevertheless, be stated that birth-associated factors clearly do not play a role, as the sharp rise in dipeptide uptake takes place well before birth. As for the postneonatal decline, changes in dietary habits that do occur in the weaning rabbit at about 10 to 20 days of age, might play a role. In fact, it has long been recognized that the diet may affect several digestive-absorptive processes, and it is conceivable that the Gly-Pro content in the diet of the weaning rabbit differs from that of the suckling animal, and that this difference may play a role. For instance, it is known that intraluminal feeding of GlyPro induces a higher Gly-Pro hydrolytic activity in the small intestinal mucosa (10).

It should also be stressed that these considerations are entirely speculative and do need proper experimental testing.

Finally, it is of interest that the rate of influx of the Gly-residue of Gly-Pro is remarkably higher as compared to the influx of free Gly. There are other reports of a kinetic advantage of dipeptide over free amino acid uptake in the adult $(4,16,18,27)$; however, in no case has this been so sizeable as in this study on the newborn animal: in the jejunum of the newborn, the uptake of peptidebound glycine is, at the concentration tested, 14 times higher than that in the free from. Thus, it appears that the intestine of the newborn animal is characterized by the physiologic capability of preferentially taking up amino acids as small peptides rather than in the free form. This physiologic finding also bears a potential nutritional significance. In fact, since free amino acids do not seem to represent the most appropriate physiologic substrate for nitrogen absorption in the youngest, it may be suggested that protein hydrolysates composed mainly of low molecular weight peptides, might be preferred as the protein components of "elemental" diets, particularly during infancy. The addition of oligopeptides might in fact offer the advantage of a lower osmotic load coupled with a fuller exploitation of the protein absorptive-digestive processes and a more efficient and fast absorption. The relative absorptive advantage of such products in man in vivo seems to be supported by studies in adults (17-28) and in infants (12).

\section{CONCLUSION}

This study (1) provides the first evidence for the existence of a dipeptide carrier system in the small intestinal brush border of fetal and newborn animals; (2) provides further, compelling evidence for its distinction from the transport systems for free amino acids; (3) suggests that the intestinal mucosa of the newborn is able to take up amino acids much more efficiently in the peptidebound than in the free form; (4) defines the developmental pattern of the dipeptide carrier system.

While further studies are needed to investigate the mechanisms responsible for the observed developmental changes in the activity of the dipeptide carrier system, it can be said that small peptides have a fundamental role in the protein digestive-absorptive processes of the youngest. This finding might prove valuable in the nutrition of neonates and infants.

\section{REFERENCES AND NOTES}

1. Addison, J. M., Burston, D., and Matthews, D. M.: Evidence for active transport of the dipeptide glycylsarcosine by hamster jejunum in vitro. Clin. Sci., 43: 907
(1972).

2. Addison, J. M., Matthews, D. M., and Burston, D.: Competition between carnosine and other peptides for transport by hamster jejunum in vitro. Clin. Sci. Mol. Med., 46: 707 (1974).

3. Adibi, S. A.: Intestinal transport of dipeptides in man: relative importance of hydrolysis and intact absorption. J. Clin. Invest., 50: 2266 (1971).

4. Adibi, S. A., and Morse, E. L.: Intestinal transport of dipeptides in man: Relative importance of hydrolysis and intact absorption. J. Clin. Invest., 50: 2266 (1971)

5. Asatoor, A. M., Cheng, B., Edwards, K. D. G., Lant, A. P., Matthews, D. M., Milne, M. D., Navab, F., and Richards, A. J.: Intestinal absorption of two dipeptides in Hartnup disease. Gut, 11: 380 (1970).

6. Chung, Y. C., Kim, Y. S., Shadchehr, A., Garrido, A., Macgregor, I. L. and Sleisenger, M. H.: Protein digestion and absorption in human small intestine. Gastroenterology, 76: 1415 (1979).

7. Cook, G. C.: Independent jejunal mechanisms for glycine and glycylglycine transfer in man in vivo. Br. J. Nutr., 30: 13 (1973).

8. Das, M., and Radhakrishnan, A. N.: Studies on the uptake of glycyl-L-leucine by strips of monkey small intestine. Indian J. Biochem. Biophys., 11: 12 (1974).

9. Deren, J. J., Strauss, E. M., and Wilson, T. H.: The development of structure and transport systems of the fetal rabbit intestine. Dev. Biol., 12: 467 (1965).

10. Dubois, R. S., Kumar, V.. and Hunka, C.: Dietary regulation of intestinal glycylproline hydrolase. Gastroenterology, 62: 745(A) (1972).

11. Fitzgerald, J. F., Reiser, S., and Christiansen. P. A.: Developmental pattern of sugar and amino acid transport in the postnatal rat small intestine. Pediatr. Res., 5: 698 (1971).

12. Guandalini, S., Palmieri, M., Di Martino, L., and Rubino, A.: Intestinal absorption of peptides and amino acids in infants Riv. Ital. Pediatr., 2: 383(A) (1976).

13. Guandalini, S., Guarino, A., and Rubino, A.: Effect of dipeptides on Na transport across rabbit's small intestine in vitro. European Intestinal Transport Group, April 1980, Southampton, England.

14. Guandalini, S., De Campora, E., Ferola, A., Migliavacca, M., Caria, C., De Gregorio, E., and Rubino, A.: Effects of malnutrition on intestinal transport of amino acids and peptides in newborn and suckling rabbits. European Society for Paediatric Gastroenterology and Nutrition, May, 1980, Capri, Italy.

15. Hellier, M. D., Holdsworth, C. D., Perret, D., and Thirumalai, C.: Intestinal dipeptide transport in normal and cystinuric subjects. Clin. Sci., 43: 659 (1972)

16. Hellier, M. D.. Radhakrishnan, A. N., Ganapathy, V., Gammon, A., and Baker, S. J.: Intestinal absorption in normal Indian and English people. Br. Med. J. 24: 186 (1976).

17. Marrs, T. C., Addison, J. M., Burston, D., and Matthews, D. M.: Changes in plasma amino acid concentrations in man after ingestion of an amino acid mixture simulating casein, and a tryptic hydrolysate of casein. Br. J. Nutr., 34 $259(1975)$.

18. Matthews, D. M.: Rates of peptide uptake by small intestine. In: Peptide transport in bacteria and mammalian gut. Ciba Foundation Symp., 4: 71 (1972).

19. Matthews, D. M., and Adibi, S. A.: Peptide absorption. Gastroenterology, 7l: 151 (1976).

20. Noren, O., Dabelsteen, E., Sjöstrom, H., and Josefsson, L.: Histological localization of two dipeptidases in the pig small intesine and liver, using immunofluorescence. Gastroenterology, $72: 87$ (1977).

21. Reiser, S., Fitzgerald, J. F., and Christiansen, P. A.: Kinetics of the accelerated intestinal valine transport in 2-day-old rats. Biochim. Biophys. Acta, 203: 351 (1970).

22. Rubino, A.: Absorption of amino acids and peptides during development. In: Milk and Lactation. Mod. Probl. Paediat. Vol. 15. p. 201 (Karger, Basel, 1975).

23. Rubino, A., Field, M., Shwachman, H.: Intestinal transport of amino acid residues of dipeptides. 1. Influx of the glycine residue of glycyl-L-proline across mucosal border. J. Biol. Chem., 246: 3542 (1971).

24. Rubino, A., and Guandalini, S.: Dipeptide transport in the intestinal mucosa of developing rabbits. In: Peptide Transport and Hydrolysis. Ciba Foundation Symposium 50: 61 (1977).

25. Rubino, A., and Guandalini, S.: (unpublished observations)

26. Schedl, H. P., Burston, D., Taylor, E., and Matthews, D. M.: Kinetics of uptake of an amino acid and a dipeptide into hamster jejunum and ileum: the effect of semistarvation and starvation. Clin. Sci., 56: 487 (1979).

27. Silk, D. B. A., Perrett, D., and Clark, M. L.: Intestinal transport of two dipeptides containing the same neutral amino acids in man. Clin. Sci. Mol. Med., 45: 291 (1973).

28. Silk, D. B. A., Clark, M. L., Marrs, T. C., Addison, J. M., Burston, D., Matthews, D. M., and Clegg, K. M.: Absorption of an amino acid mixture simulating casein and of an enzymic hydrolysate simulating casein prepared for oral administration to normal adults. Br. J. Nutr., 33: 95 (1975).

29. Sjöstrom, H., Noren, O., and Josefsson, L.: Purification and specificity of pig intestinal prolidase. Biochim. Biophys. Acta, 327: 457 (1973)

30. States, B., and Segal, S.: Developmental aspects of cystine transport in rat intestinal segments. Biochim. Biophys. Acta, 163: 154 (1968).

31. Sugiyama, K., Yasumoto, K., and Mitsuda, N.: Effect of semistarvation on kinetic parameters for the in vitro accumulation and transport of $\mathrm{L}$-leucine in rat small intestine. Agric. Biol. Chem., 41: 1969 (1977).

32. Requests for reprints should be addressed to: Dr. Stefano Guandalini, Cattedra di Puericultura, 2nda Facoltà di Medicina dell'Università, Via S. Pansini, 5 80131 , Napoli, Italy.

33. Part of this study was presented in preliminary form at the Ciba Foundation Symposium 50, London, November 1976

34. Received for publication October 7, 1980

35. Accepted for publication April 3, 1981. 